\title{
A new test cell for the evaluation of thermo-physical performance of facades building components
}

\author{
Giuseppina Alcamo ${ }^{\mathrm{a}, *}$ and Maurizio De Lucia ${ }^{\mathrm{b}}$ \\ ${ }^{a}$ Department of Architecture DIDA, University of Florence, San Niccolò 93, 50125 Florence, Italy; \\ ${ }^{b}$ Department of Industrial Engineering, University of Florence, via Santa Marta 3, \\ 50139 Florence, Italy
}

(Received 29 January 2013; final version received 14 April 2013)

\begin{abstract}
How do the new building façade components work under real climatic conditions? The question is especially related to the dynamic behaviour of new and complex components such as the ventilated walls, the Phase Change Material, or others that use nano-technologies and aerogels. One of the objectives of the undergoing research activity, named Abitare Mediterraneo, is the evaluation of their thermal behaviour through the use of an outdoor test cell, in order to reproduce under the real external conditions the performance of these components, and consequently define the main thermal parameters such as the attenuation factor and the thermal inertia, characterising the component; moreover, the results will be used to write new algorithms for dynamic simulation tools allowing an appropriate evaluation of the complex behaviour of the whole building at a real scale condition. The new test cell is realised in Florence, Italy. The design starts from the study of the experience of the existing PASSYS test cells, overtaking the main weakness of the building structure and of the heat-flux sensors.
\end{abstract}

Keywords: test cell; dynamic performance of façade building components; Mediterranean climate; heat-flux sensors

\section{Introduction}

The Mediterranean climate represents a great opportunity for architects in order to define the most appropriate design and technological solutions to fulfil the users' expectations.

The traditional building design rules provide the first inspiration in terms of shapes, and solutions', giving the needed bioclimatic strategies. The idea to conjugate tradition and innovation is a substantial stimulating challenge; today, several new energy-saving components can be adopted, but to demonstrate their energy efficiency they must be tested in outside, dynamic and real weather conditions. A new test cell was designed at the University of Florence with the aim to dynamically evaluate thermo-physical properties and day-lighting distribution under real sky conditions.

A special effort has been devoted to the measurements of heat fluxes in the opinion of the authors that represent the most significant improvement compared to the solutions presented and used to date.

*Corresponding author. Email: giuseppina.alcamo@unifi.it 